\title{
Inmigración y Pensiones: ¿Qué sabemos?
}

\author{
J. Ignacio Conde-Ruiz \\ FEDEA y UCM \\ Juan F. Jimeno \\ Banco de España \\ Guadalupe Valera \\ Universidad Pablo de Olavide de Sevilla
}

\section{Resumen}

Este artículo ofrece una panorámica de la literatura académica sobre los efectos de la inmigración en los gastos e ingresos de los sistemas de pensiones. Un argumento frecuente es que el aumento (selectivo) de la población inmigrante ayuda a medio plazo al equilibrio financiero del sistema de pensiones. Si bien, la entrada de inmigrantes en edad de trabajar retrasa el aumento de la tasa de dependencia derivado del envejecimiento de la población, al generar derechos a pensiones futuras, traslada el problema financiero a la siguiente generación. En definitiva, a largo plazo, en la medida en que los inmigrantes adopten las pautas demográficas de los nativos, la inmigración no resuelve los problemas financieros que puedan estar implicitos en los sistemas de pensiones en vigor.

Palabras clave: sistemas de pensiones de reparto y prestación definida, inmigración, tasa de dependencia, tasa de empleo, educación.

Clasificación JEL: H55, F22, J21, J24

\begin{abstract}
This article offers a survey of the literature that deals with the effects of immigration on the financial flows of pension security systems. It is usually argued that a selective increase of immigration flows helps to the financial equilibrium of the system on the medium term. The arrival of immigrants in working age improves the financial sustainability since delays the rise of the dependency rate caused by population ageing, but those immigrants also generate the right to future pensions. Over the long run, as immigrants adopt the demographic patterns of the native population, immigration cannot solve the financial problems that may be embedded in the current pension systems.
\end{abstract}

Key Words: defined benefit PAYGO pension system, immigration, dependency ratio, employment rate, education.

JEL Classification: H55, F22, J21, J24

\section{Introducción}

En los últimos años, tanto las políticas de inmigración como las políticas de pensiones han generado un gran debate en los países occidentales, aunque generalmente han sido analizadas de forma independiente. Por tanto, no ha de sorprendernos que 
su análisis conjunto pueda resultar tan excitante como polémico. Existe una amplia literatura dedicada a estudiar el efecto de los flujos de inmigración sobre la economía receptora en su conjunto, si bien el principal esfuerzo investigador se ha centrado en analizar como afectan al mercado de trabajo. Más recientemente, el debate sobre la sostenibilidad financiera de los sistemas de pensiones de los países occidentales, mayoritariamente de reparto, ha desplazado el esfuerzo investigador hacia el análisis de los efectos de la inmigración sobre los sistemas de pensiones.

El envejecimiento experimentado por la población occidental, sobre todo en Europa, debido fundamentalmente a un aumento espectacular de la esperanza de vida (más de 8 años en las últimas dos décadas) unido a las bajas tasas de natalidad registradas en Europa a partir de los años 1970s, (en el caso de España a partir de principios de la década de los años 1980s), tiene un impacto claro y directo sobre los sistemas de pensiones de reparto, pues tiende a aumentar la proporción de jubilados mientras que reduce la proporción de trabajadores en activo. En este contexto, no son pocos los que se han aventurado a sostener que sólo abundantes flujos de inmigración podrían restaurar una relación entre trabajadores en activo y jubilados que fuera compatible con el equilibrio financiero de los actuales sistemas de pensiones en estos países.

Este artículo ofrece una panorámica de algunas aportaciones tanto teóricas como empíricas sobre esta cuestión. Para ello, se analizan los canales mediante los cuales la inmigración puede tener un impacto sobre el equilibrio financiero de un sistema de pensiones de reparto. La estructura del artículo es la siguiente: la Sección 2 resume las principales aportaciones teóricas y empíricas sobre la relación entre inmigración y políticas redistributivas en el sentido más amplio, incluyendo aquellas contribuciones de la Economía Política que señalan que las decisiones sobre los sistemas fiscales y programas de gasto social están condicionadas por el grado de desigualdad económica; la Sección 3 se concentra en analizar los determinantes del gasto en pensiones y como la inmigración puede influir sobre ellos; por último, la Sección 4 contiene algunos comentarios finales.

\section{Efectos Redistributivos de la Inmigración}

Tradicionalmente, la teoría económica ha analizado los movimientos migratorios desde distintos enfoques, y sólo en los últimos años se han empezado a relacionar con los sistemas de pensiones; este interés surge precisamente cuando la sostenibilidad financiera y el carácter redistributivo de los actuales sistemas de pensiones está sometida a un intenso debate en los países occidentales destino de los principales flujos migratorios. Por este motivo, creemos que antes de pasar a exponer los trabajos que se han centrado en el efecto de la inmigración sobre las pensiones, es necesario exponer brevemente los trabajos dedicados a analizar los efectos redistributivos de la inmigración.

La mayoría de los países occidentales tienen estructurado un sistema de bienestar social compuesto por una serie de programas de redistribución de la renta y segu- 
ridad social. Como es lógico, la evolución demográfica tiene importantes implicaciones en las políticas redistributivas del sector público. Así, la llegada de inmigrantes, afecta tanto a los ingresos como a los gastos públicos, de forma que el saldo neto de la inmigración depende no sólo de las particularidades del Estado del Bienestar sino también de las características (edad, nivel educativo y estructura familiar) del flujo de inmigrantes. Normalmente, los Estados del Bienestar modernos redistribuyen renta de individuos ricos a individuos pobres a través de trasferencias de renta o en especie, convirtiéndose así en destinos muy atractivos para la inmigración, principalmente de baja cualificación. De esta forma, los programas redistributivos, implícita o explícitamente, dan lugar a un fenómeno de selección adversa: los beneficiarios netos se sienten atraídos por países con alto grado de redistribución, mientras que los contribuyentes netos se sienten repelidos. Esta idea de la inmigración inducida por la redistribución, se ha explorado profundamente en literatura clásica sobre federalismo fiscal (Sitgler (1957), Oates (1968), Mirrless (1982) y Musgrave (1969)) aplicándose posteriormente en un contexto internacional.

Siguiendo esta línea de investigación, Wildasin (1994) utiliza un modelo de equilibrio general con dos países y libre circulación de trabajadores, para analizar las políticas de redistribución que transfieren renta entre los propietarios de los factores de producción fijos y los trabajadores en una determinada región. Caracteriza una frontera de posibilidades de redistribución y las posibles combinaciones de distribución de renta por medio de una política de impuestos y transferencias. Muestra que en presencia de inmigración se pueden alcanzar asignaciones Pareto inferiores en el país de destino de la inmigración si los inmigrantes son beneficiarios de transferencias y, por lo tanto, imponen una carga fiscal sobre la sociedad. Una clara implicación de este resultado es que los inmigrantes ya residentes en el país receptor podrían desear la limitación de la inmigración futura. Esta idea de que existe una resistencia a la entrada de inmigrantes poco cualificados al ser potencialmente receptores netos de los programas del Estado del Bienestar en el corto plazo (análisis estático), también se encuentra en el trabajo de Razin y Sadka (1995). Se podría concluir entonces que en modelos estáticos y puramente redistributivos existirá una oposición por parte de los nativos a la entrada de inmigrantes poco cualificados.

Müller (1999), intenta explicar por qué desde los años 1950s y 1960s las políticas migratorias se han vuelto cada vez más restrictivas con respecto a la entrada de trabajadores poco cualificados. En contraposición a la idea de que este aumento del proteccionismo se debe a las dificultades macroeconómicas y al aumento del desempleo — sobre todo en Europa — , muestra que no existe evidencia empírica de que la inmigración afecte al desempleo. Para ello enlaza el efecto de la inmigración sobre el mercado de trabajo y sus efectos redistributivos, incluyendo de forma explícita la redistribución de la renta. Desarrolla un modelo con un mercado de trabajo dual con discriminación hacia los inmigrantes, definida no como menor salario por el mismo trabajo, sino como una restricción de sus posibilidades de promoción a trabajos más cualificados y por consiguiente con mayor salario. Müller (1999) 
sostiene que si bien la ganancia total de la entrada de inmigrantes poco cualificados resulta positiva (criterio utilitarista), un análisis de bienestar completo ha de tener en cuenta como afecta dicha entrada a la desigualdad de la renta. Y precisamente muestra como la discriminación laboral hacia los inmigrantes genera un aumento en la desigualdad de la renta de tal calibre que criterios de bienestar social, que tuvieran en cuenta la desigualdad, desaconsejarían la entrada de inmigrantes poco cualificados.

Por el contrario, si consideramos un entorno dinámico con transferencias intertemporales (como las pensiones), Razin y Sadka (1999) demuestran que la entrada de inmigrantes poco cualificados podría resultar beneficiosa para todos los nativos (ricos y pobres). En su modelo, los inmigrantes no cualificados también son beneficiarios netos del sistema de pensiones, pero ello no impide que todas las generaciones nativas (jóvenes y mayores), independientemente de su nivel de renta mejoren. Este resultado se obtiene al considerar todo el ciclo vital de los inmigrantes, observando que no son beneficiarios netos en todos los periodos: concretamente en el periodo de llegada, son contribuyentes netos a las finanzas públicas, de forma que los nativos jubilados de ese periodo reciben mayores pensiones. En el siguiente periodo esos inmigrantes han generado el derecho a recibir una pensión, pero aseguran la financiación de las mismas, aumentando la población de jóvenes con sus hijos, que para entonces ya son nativos. Por lo tanto, en un contexto dinámico, Razin y Sadka (1999) concluyen que en una sociedad democrática el equilibrio político resultante de una votación podría ser pro-inmigración (cuanta más mejor).

La literatura empírica, por su parte, también ha analizado los efectos redistributivos de la inmigración. Varios trabajos cuantitativos realizados para la economía de Estados Unidos demuestran que el coste o el beneficio fiscal de la inmigración en su conjunto resultan muy bajos (Borjas y Trejo (1991), Borjas (1994)). El panorama cambia drásticamente si analizamos las ganancias netas por edad o nivel educativo. Storesletten (2000) desarrolla un modelo de equilibrio general con generaciones solapadas a la Auerbach y Kotlikoff (1987) donde los inmigrantes son heterogéneos en habilidad, edad y el año de entrada — legal — en Estados Unidos. El modelo de generaciones solapadas permite cuantificar el efecto de los inmigrantes a través de su ciclo vital: i) la entrada de inmigrantes en edad de trabajar aumenta los ingresos fiscales a través del impuesto sobre la renta, reduciendo tanto la deuda como el gasto del gobierno por trabajador; no obstante ii) cuando dichos inmigrantes se jubilan los efectos anteriores tienen precisamente el signo contrario. Lo interesante del artículo es que también cuantifica el efecto de equilibrio general sobre el presupuesto del gobierno. Bajo el supuesto de que no se produce salida de capitales, demuestra que al aumentar la fuerza de trabajo la ratio trabajo-capital también aumenta y como consecuencia disminuye el salario de equilibrio y aumenta el tipo de interés real. La modificación de los precios relativos de equilibrio debido a la inmigración tiene fuertes implicaciones cuantitativas sobre la política fiscal: la subida de los tipos de interés aumenta el servicio de la deuda pública y el descenso de los salarios disminuye los ingresos impositivos por trabajador. 
Finalmente, estudia en términos presentes, la ganancia neta para el gobierno de admitir un inmigrante adicional en función de sus características. Lógicamente se observa que cuantitativamente los inmigrantes jóvenes y con alta habilidad son contribuyentes netos a las arcas del estado, mientras que los inmigrantes sin cualificación y los jubilados representan un alto coste fiscal (estos resultados coinciden con Lee y Miller (1997)).

El Estado del Bienestar de Estados Unidos es muy distinto al de los otros países occidentales. Estados Unidos tiene un sector público pequeño (22\% del PIB), impuestos bajos, poco desempleo y un sistema de seguro público relativamente limitado. Por el contrario los Estados del Bienestar europeos son grandes (en media alcanzan el $41 \%$ del PIB), impuestos elevados, mayor desempleo y además cuentan con generosos sistemas de asistencia publica. Por este motivo, los análisis cuantitativos realizados para los países europeos (escasos todavía) dan resultados distintos a los obtenidos para Estados Unidos. Storesletten (2003) calcula la ganancia pública neta de un nuevo inmigrante, definida como el valor descontado de los pagos impositivos futuros menos las transferencias, incluyendo el coste y las contribuciones de los hijos futuros para la economía sueca ${ }^{1}$. Bajo esta óptica, un nuevo inmigrante se ve implícitamente como una inversión pública. Las implicaciones del modelo sugieren que la ganancia pública neta es potencialmente alta si los inmigrantes cuentan entre 20 y 30 años en el momento en que entran en Suecia. Por el contrario, inmigrantes mayores de 50 años o menores de 10 suponen un coste neto sustancial. Es decir, en términos cualitativos los resultados obtenidos para Suecia y Estados Unidos resultan muy similares, especialmente si nos fijamos en la distribución por edad. No obstante, las magnitudes son diferentes, obteniendo Estados Unidos mayores ganancias potenciales.

Respecto a la utilización que la población inmigrante hace del Estado del Bienestar, $\mathrm{Hu}$ (1997) encuentra que la mayor diferencia entre nativos e inmigrantes en el disfrute de los distintos programas se concentra en la población anciana. El resultado más importante para la política de inmigración es que los inmigrantes que en el momento de entrada cuentan con más de 55 años tienen una probabilidad significativamente mayor de acogerse a los programas incluidos en el Estado del Bienestar que los inmigrantes que entraron en edad de trabajar, al alcanzar esa misma edad. Así, sorprendentemente, la variable más relevante para explicar la diferencia en el uso de los programas del bienestar es la edad de entrada en el país y no tanto los niveles de ingresos de los inmigrantes, inferiores a los de los nativos.

Por último, es interesante realizar un recorrido por los distintos análisis positivos del efecto redistributivo de la inmigración. Los análisis positivos suponen que el nivel y diseño de los programas de redistribución de un Estado del Bienestar es el re-

Analizar el impacto de la inmigracion sobre el Estado del Bienestar sueco es muy interesante, pues Suecia no sólo cuenta con un Estado del Bienestar grande sino que ademas absorbe una gran flujo de inmigrantes (desde 1989 entran anualmente en Suecia un 0,67 \% de la poblacion total, aproximadamente). 
sultado de un equilibrio político económico que agrega las preferencias de los distintos individuos en un vector de política. Por lo tanto la entrada de inmigrantes en un país puede potencialmente aumentar o disminuir el apoyo político de un determinado programa de redistribución. Asumiendo que los inmigrantes obtienen el derecho a votar en el momento de entrar en el país, Razin y Sadka (1997) se preguntan si la entrada de inmigrantes no cualificados genera una mayor demanda de redistribución, y en consecuencia, un aumento de la presión fiscal. Sorprendentemente, la respuesta no es tan sencilla como parecería a priori, pues se producen dos efectos contrapuestos: por un lado los inmigrantes de renta baja, beneficiarios netos de los programas de redistribución, unirán sus fuerzas con los nativos pobres para aumentar la generosidad total de las transferencias, y por ende los impuestos; por otro lado, la redistribución se vuelve más costosa para la población nativa, pues ahora el gasto total debe repartirse con los inmigrantes.

En esta misma línea, Razin, Sadka y Swagel (2002) presentan una extensión del modelo seminal de Metzler y Richard (1981) que incluye la entrada endógena de inmigrantes. Sorprendentemente, demuestran que la entrada de inmigrantes poco cualificados podría generar una disminución en la redistribución de la renta. De nuevo, la intuición de este resultado reside en el aumento del número de receptores de los programas redistributivos (los inmigrantes), que provoca que el votante mediano, a pesar de ser relativamente más pobre, obtenga una transferencia per capita menor para el mismo nivel impositivo.

Ortega (2004) utiliza un modelo dinámico de economía política donde los individuos votan sobre la política de inmigración. Lo interesante de este artículo reside principalmente en la decisión de voto de los trabajadores nativos muy cualificados. En el corto plazo, estos trabajadores se benefician de la entrada de inmigrantes poco cualificados debido a su complementariedad laboral. No obstante, en el largo plazo, al obtener derecho al voto, los inmigrantes demandarán una mayor redistribución de la renta y por lo tanto un aumento de la carga impositiva.

Además, es interesante resaltar que el modelo de Razin y Sadka (2000), analizado anteriormente, concluye que cuando el juego de redistribución es intertemporal, el equilibrio político resultante es pro-inmigración formándose una coalición ganadora de jubilados (más inmigración implica pensiones medias más altas) y trabajadores cualificados (salarios para los trabajadores cualificados y retornos del capital más altos por la complementariedad con los trabajadores poco cualificados). Kemnitz (2003) en un modelo con un mercado de trabajo competitivo y donde el país de acogida tiene un Estado del Bienestar que protege tanto a los desempleados como a los pensionistas, obtiene que la entrada de inmigrantes genera una ganancia de bienestar para la población nativa en su conjunto.

Por último, Tamura (2006) matiza el hecho de que los nativos poco cualificados siempre estén en contra de la inmigración y en un modelo con un Estado del Bienestar que redistribuye hacia individuos pobres e individuos mayores encuentra situaciones donde incluso los trabajadores poco cualificados estarían dispuestos a apoyar la entrada de inmigrantes de su mismo nivel de cualificación. 


\section{Inmigración y pensiones}

Hasta ahora hemos repasado brevemente la amplia literatura existente dedicada a estudiar el efecto de los flujos de inmigración sobre los estados de bienestar en su conjunto de los países receptores. Sin embargo, más recientemente, el debate sobre la sostenibilidad financiera de los sistemas de pensiones de reparto de los países occidentales ha desplazado el esfuerzo investigador hacia el análisis de los efectos de la inmigración sobre dichos sistemas.

En concreto, el debate actual se centra en el envejecimiento de la población y su repercusión sobre la sostenibilidad financiera de los sistemas de pensiones de reparto y prestación definida. Este envejecimiento poblacional tiene un impacto directo sobre los sistemas de seguridad social, pues tiende a aumentar la proporción de jubilados mientras que reduce la proporción de trabajadores en activo. Dado que cada vez hay más individuos que cobran del sistema y menos individuos que contribuyen a él, una sociedad envejecida no será capaz de hacer frente a los beneficios comprometidos con los trabajadores futuros, a no ser que o bien se reforme el sistema o bien se incremente la carga impositiva de los trabajadores. Al mismo tiempo, en algunos foros se apuesta por posponer las reformas y apostar por la inmigración como único mecanismo para alcanzar el equilibrio presupuestario de la seguridad social en el futuro. El objetivo de esta sección es recorrer las principales aportaciones académicas que analizan cuál es el impacto de la inmigración sobre la sostenibilidad financiera de los sistemas de seguridad social de reparto.

Una forma sencilla de analizar la relación entre el envejecimiento de la población y el gasto en pensiones consiste en analizar los determinantes fundamentales del gasto agregado en pensiones. Para ello, al igual que Jimeno (2002a y 2002b) descomponemos el gasto en pensiones como porcentaje del PIB en los siguientes tres factores (factor demográfico, factor mercado de trabajo y factor institucional):

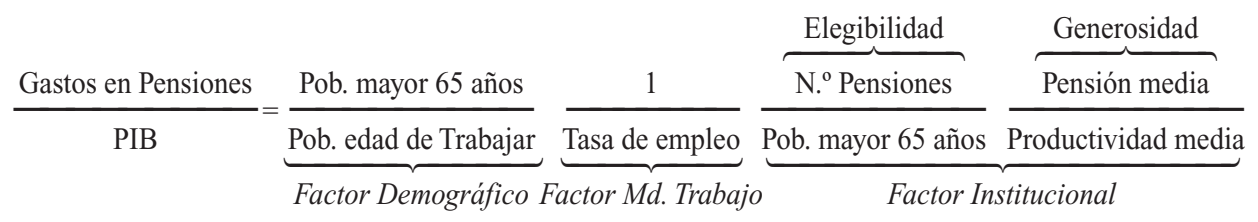

A continuación, a través de los principales trabajos de investigación, analizaremos el efecto de la inmigración sobre cada uno de los tres factores que determinan el gasto en pensiones futuro como porcentaje del PIB.

\subsection{Inmigración y Demografía}

El Factor Demográfico depende de la evolución demográfica y es equivalente a la tasa de dependencia de los trabajadores mayores. La tasa de dependencia de las per- 
sonas mayores se define como el cociente entre el número de individuos mayores de 64 años y el número de individuos con edades comprendidas entre los 20 y los 64 años. De acuerdo con las proyecciones de la OCDE, la ratio de dependencia de las personas mayores para la media de los países de la OCDE se incrementará del 23,8 en el año 2000 al 49,9 en el año 2050. En el gráfico 1 vemos que entre los años 1995 y 2050 las tasas de dependencia de las personas mayores de 60 años estimadas por Eurostat para la Unión Europea se pueden multiplicar por dos, siendo España, junto con Italia e Irlanda los países de la Unión Europea que se encontrarán con las tasas de dependencia más elevadas.

Para entender mejor el efecto de la inmigración sobre la tasa de dependencia, pensemos en un modelo muy sencillo con tres generaciones como el que se recoge en el gráfico 2. En cada momento de tiempo la estructura poblacional esta compuesta por individuos mayores, individuos maduros y jóvenes. Actualmente, la estructura poblacional de la mayoría de los países occidentales tiene forma de rombo. Esta estructura de la población implica que existen varios trabajadores potenciales por cada jubilado, y por lo tanto, una tasa de dependencia relativamente baja. En un contexto de progresivo envejecimiento de la población, la estructura poblacional de la mayoría de los países occidentales tendrá forma de $\mathrm{T}$ en el año 2050, lo que supone una tasa

\section{GRÁFICO 1}

TASA DE DEPENDENCIA DE LOS MAYORES (\% PERSONAS MAYORES DE 60 AÑOS CON RESPECTO A LA POBLACIÓN EN EDAD DE TRABAJAR)

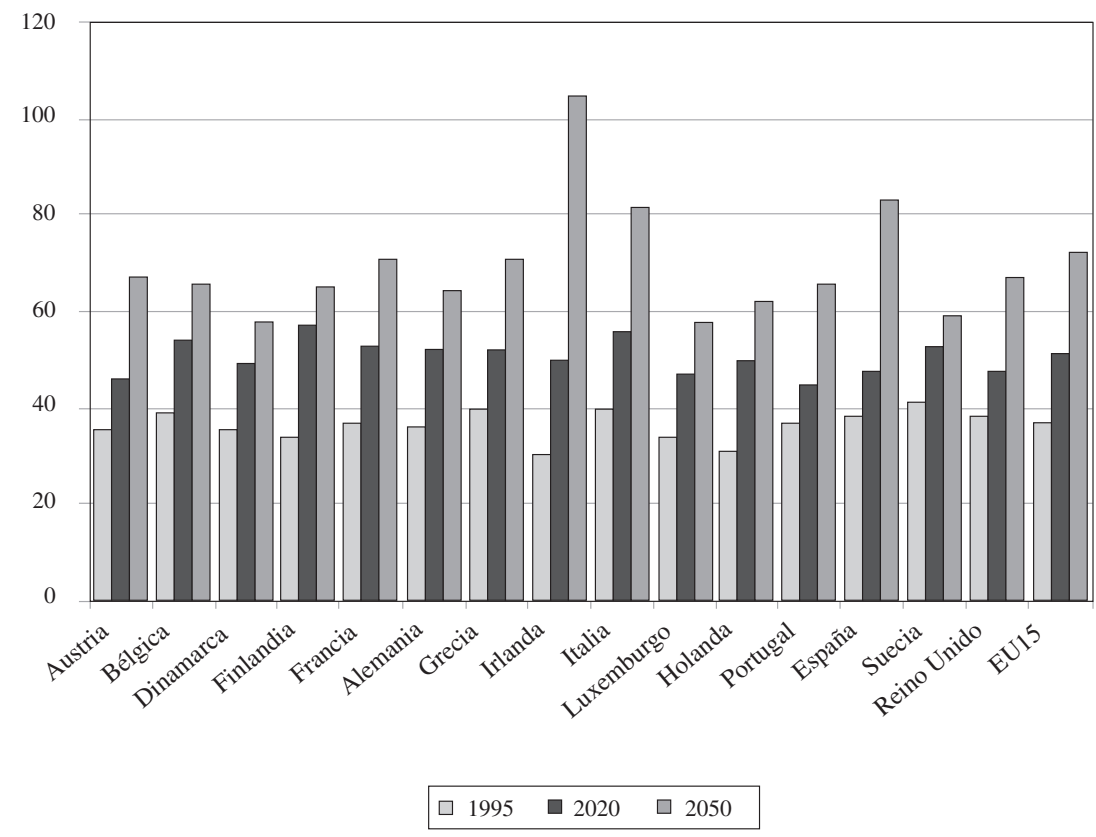

Fuente: Eurostat 


\section{GRÁFICO 2}

\section{SITUACIÓN INICIAL. DUPLICACIÓN DE LA TASA DE DEPENDENCIA}

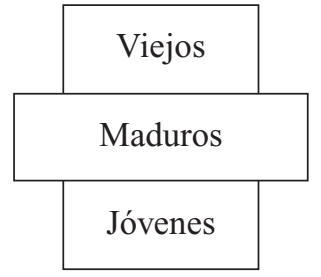

Periodo t

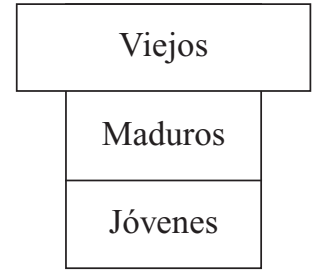

Periodo $\mathbf{t}+1$

T. Dependencia ${ }_{t}=\frac{\text { viejos }_{t}}{\text { trabajadores }_{t}}=\frac{1}{3}$

T. Dependencia $a_{t+1}=\frac{\text { viejos }_{t+1}}{\text { trabajadores }_{t+1}}=1$

de dependencia tan alta que pone en peligro la sostenibilidad financiera de los sistemas de pensiones de reparto y prestación definida.

Frente a este progresivo envejecimiento de la población de los países occidentales, la tasa de fecundidad de los países en vías desarrollo continúa aumentando. Por lo tanto, parece abrirse una «ventana de oportunidad» para que la llegada de inmigrantes permita reducir las tasas de dependencia que se prevén para el futuro. Pero, ¿puede la inmigración por si sola evitar que se disparen las tasas de dependencia? Gráficamente, esta cuestión equivale a preguntarse si la inmigración es capaz de modificar la evolución de la pirámide poblacional de manera que no adquiera forma de T. En concreto, el efecto de la inmigración sobre la composición por edades de la población nativa depende de tres factores: i) la edad de los inmigrantes, ii) sus pautas de fecundidad; y iii) la tasa de retorno a sus países de origen.

Desde el punto de vista de la toma de decisiones políticas, el elemento clave es la edad de los inmigrantes en el momento de entrada en el país receptor. Supongamos que los individuos jóvenes son los hijos de los individuos maduros y además que los inmigrantes adquieren unas pautas de comportamiento igual a la de los nativos. En los gráficos 3 y 4 se observa que el efecto de la inmigración sobre la tasa de dependencia futura depende de la edad del inmigrante en el momento de entrar: en el gráfico 3 analizamos la evolución de la tasa de dependencia futura si entran inmigrantes maduros, mientras que en el gráfico 4 vemos qué ocurre cuando entran inmigrantes jóvenes. En primer lugar, la entrada de inmigrantes jóvenes afecta en mayor medida a la tasa de dependencia futura que la entrada de inmigrantes maduros. La causa es evidente: la tasa de dependencia se dispara precisamente, porque la generación que se jubilará en el futuro es mucho más numerosa que la que se incorporara a trabajar. En nuestro sencillo modelo, la tasa de dependencia aumenta en $t+1$ en porque la generación madura en $t$ (es decir, la generación mayor en $t+1$ ) es mayor que la generación madura en $t+1$ (la generación joven en $t$ ). Si los inmigrantes que llegan en $t$ corresponden a la generación madura, no harán más que aumentar aún más el numero de individuos mayores en $t+1$, aumentando, en consecuencia, la tasa de dependencia. Por 


\section{GRÁFICO 3}

ENTRADA DE INMIGRANTES DE EDAD MADURA

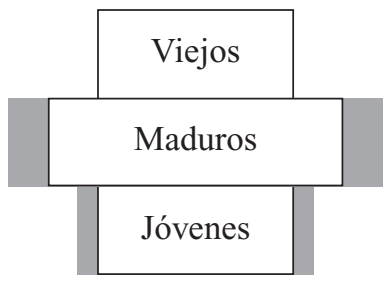

Periodo t

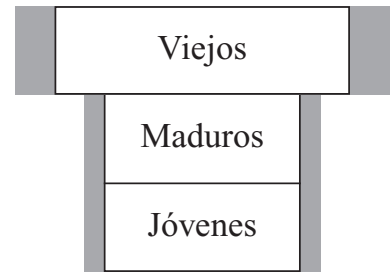

Periodo t+1

T. Dependencia ${ }_{t}=\frac{\text { viejos }_{t}}{\text { trabajadores }_{t}}=\frac{1}{4}$

T. Dependencia $a_{t+1}=\frac{\text { viejos }_{t+1}}{\text { trabajadores }_{t+1}}=1$

\section{GRÁFICO 4}

\section{ENTRADA DE INMIGRANTES DE EDAD JOVEN}

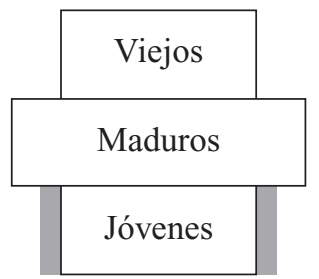

Periodo t

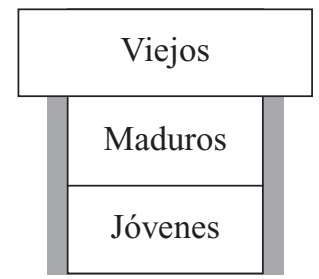

Periodo t+1

T. Dependencia ${ }_{t}=\frac{\text { viejos }_{t}}{\text { trabajadores }_{t}}=\frac{3}{7}$

$\mathrm{T}$. Dependencia $\mathrm{D}_{t+1}=\frac{\text { viejos }_{t+1}}{\text { trabajadores }_{t+1}}=\frac{1}{3}$

tanto, si el objetivo es ralentizar el incremento de la tasa de dependencia futura resulta necesario que la estructura de la población inmigrante sea complementaria de la estructura poblacional nativa.

Claramente, la distribución de inmigrantes óptima por edad depende de la estructura poblacional nativa del país en cuestión. Si consideramos el caso de España, la problemática de la sostenibilidad financiera del sistema de pensiones es especialmente preocupante dadas sus bajas tasas de fecundidad a partir de la primera década de los años 1980s y por el espectacular aumento en la esperanza de vida. Si examinamos las pirámides de población nativa e inmigrante del año 2001 según el Censo de Población y Viviendas (2001), se advierte una gran similitud entre ambas, en el sentido de que las mayores cohortes de la población nativa coinciden con las mayores cohortes de la población inmigrante. Si el objetivo político es contrarrestar el crecimiento de la tasa de dependencia, la entrada de inmigrantes en edad madura resulta claramente poco deseable. 
Finalmente, merece la pena considerar las diferencias entre la pirámide poblacional de los inmigrantes comunitarios y los extracomunitarios. Los gráficos 5 a 8 muestran, por un lado, la estructura poblacional de los inmigrantes comunitarios, envejecida debido a que un gran porcentaje de comunitarios viene a España ya jubilado atraídos por un clima benigno y, por otro, la estructura de los extracomunitarios, madura pues se trata de individuos que entran en España para trabajar. Esta diferencia resultará importante cuando analicemos el factor institucional y concretamente la elegibilidad, pues los inmigrantes que entran en España después de jubilarse reciben la pensión de las arcas de su país de origen.

\subsection{Inmigración y Mercado de Trabajo}

El Factor Mercado de Trabajo es la inversa de la tasa de empleo, por lo tanto cuanto mayor sea la tasa de empleo más pequeño será dicho factor y menor será el gasto en pensiones como porcentaje del PIB. La teoría económica ${ }^{2}$ identifica la estructura

\section{GRÁFICO 5 \\ PIRÁMIDE DE POBLACIÓN PARA LA POBLACIÓN NATIVA (ESPAÑA)}

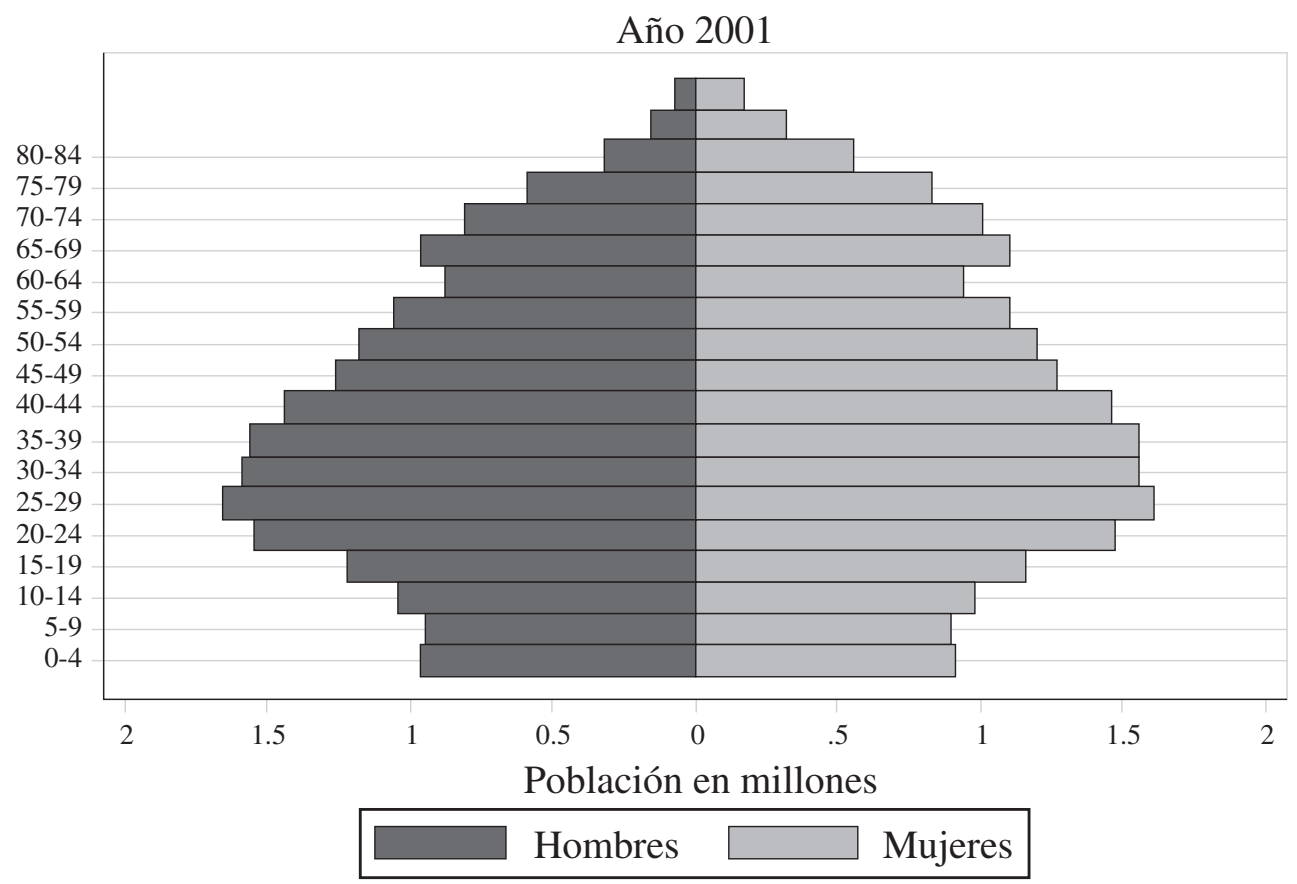

Fuente: Censo de Población y Viviendas 2001.

2 Borjas (1994 y 1999) recoge las aportaciones principales sobre el efecto de la inmigración en el mercado de trabajo de los paises de acogida. 


\section{GRÁFICO 6}

\section{PIRÁMIDE DE POBLACIÓN PARA LA POBLACIÓN INMIGRANTE EN ESPAÑA}

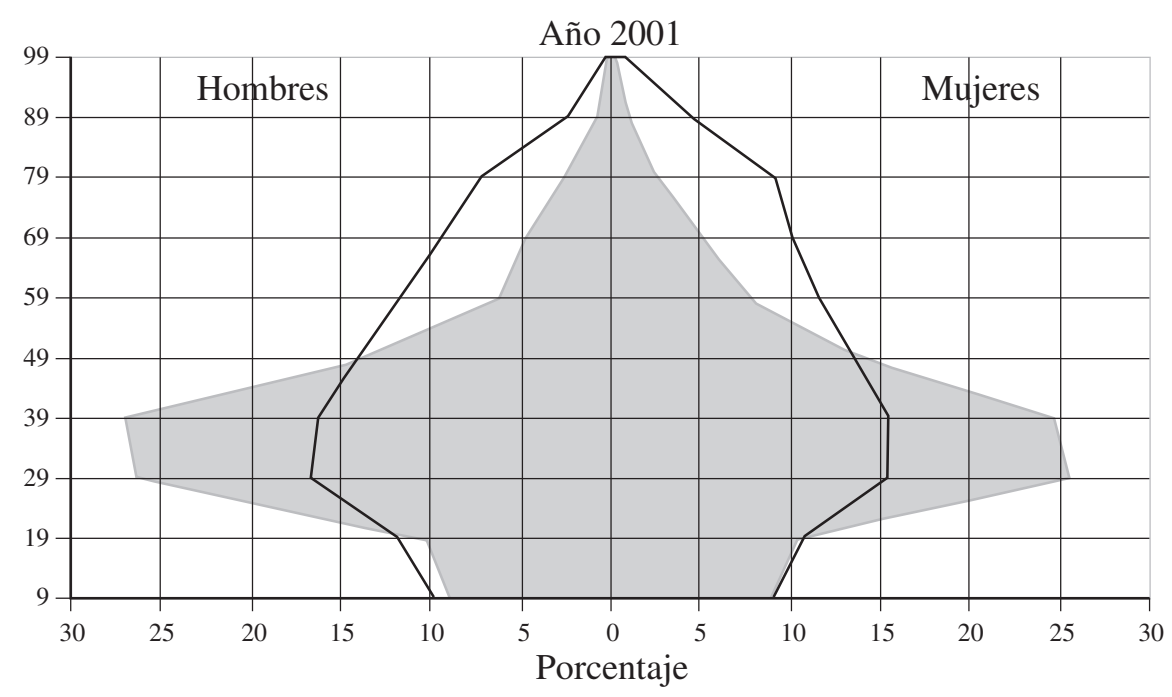

Pirámide de la población inmigrante

Perfil de la población española

Fuente: Censo de Población y Viviendas 2001.

\section{GRÁFICO 7}

PIRÁMIDE DE POBLACIÓN PARA LA POBLACIÓN INMIGRANTE (COMUNITARIOS)

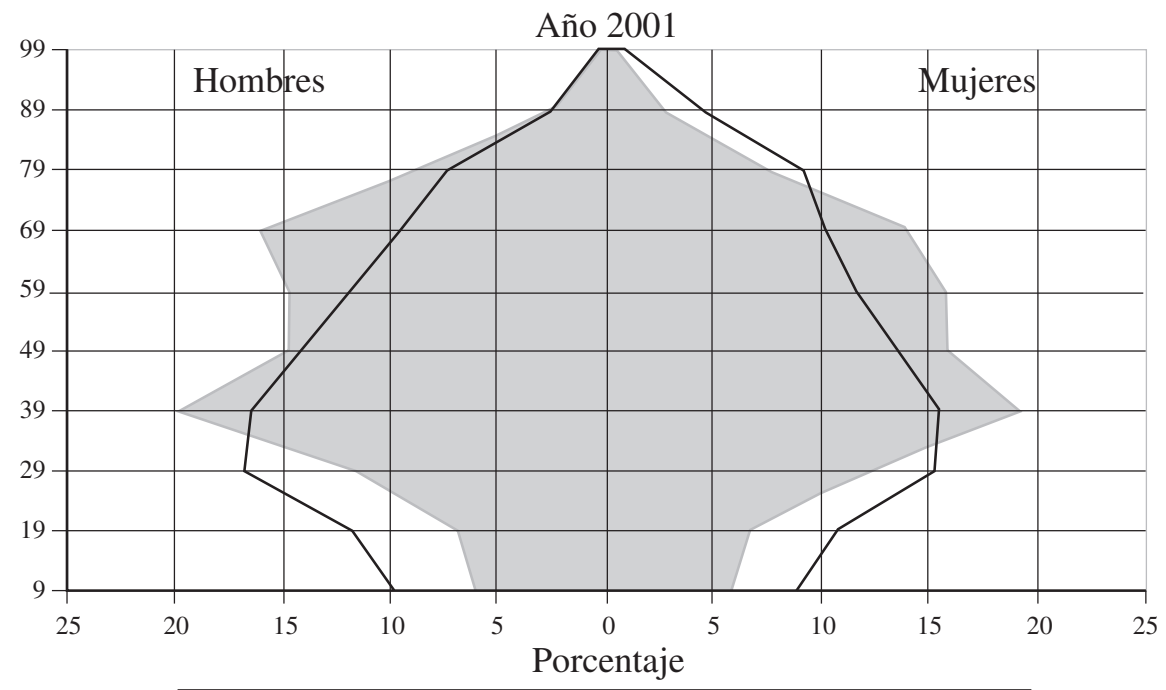

Pirámide de la población inmigrante comunitaria

Perfil de la población española

Fuente: Censo de Población y Viviendas 2001. 


\section{GRÁFICO 8}

PIRÁMIDE DE POBLACIÓN PARA LA POBLACIÓN INMIGRANTE (EXTRACOMUNITARIOS)

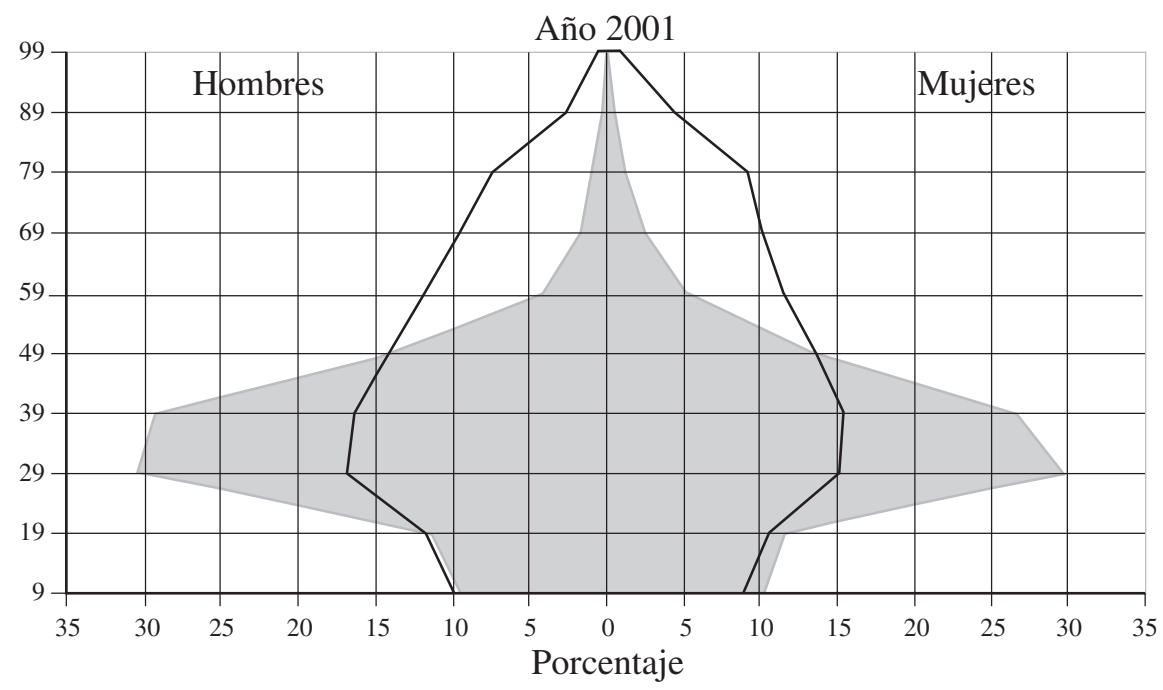

Pirámide de la población inmigrante extra comunitaria

Perfil de la población española

Fuente: Censo de Población y Viviendas 2001.

educativa de la población inmigrante como el elemento clave para comprender el impacto de la inmigración sobre los mercados de trabajo de los países de acogida. En un modelo muy sencillo con trabajo homogéneo, la entrada de inmigrantes amplía la oferta de trabajo y como consecuencia disminuye el salario y aumenta el empleo, al menos en el corto plazo. Si suponemos ahora que el factor trabajo es heterogéneo, los efectos son más complejos y la estructura educativa o por habilidad de las dos poblaciones (inmigrante y nativa) juega un papel clave. En concreto, si el mercado de capitales es perfectamente elástico podemos considerar tres posibilidades: i) si el nivel formativo de ambas poblaciones es idéntico, la entrada de inmigrantes no tiene ningún efecto sobre el mercado de trabajo; ii) si los inmigrantes se hallan menos cualificados que la población nativa, entonces el salario de los trabajadores poco cualificado disminuye mientras que el salario de los trabajadores cualificados aumenta; iii) si los inmigrantes están más cualificados, entonces se produce el efecto inverso. Consecuentemente, si el nivel de cualificación de inmigrantes y nativos difiere — casos ii) y iii) - el efecto total sobre la tasa de empleo no es monótono y depende de las elasticidades.

Existe una literatura empírica muy extensa (resumida en Borjas (1994)) que analiza el impacto de los inmigrantes sobre la tasa de desempleo (o las oportunidades de empleo de la población nativa). Todos los trabajos coinciden en afirmar que el impacto de la inmigración sobre la tasa de desempleo es muy débil. Por lo tanto, podríamos 
concluir que el impacto de la inmigración sobre el Factor Mercado de Trabajo definido en la ecuación (1) es prácticamente nulo.

En los gráficos 9 y 10 se compara la estructura educativa de la población nativa española y la de la población inmigrante. Las conclusiones más relevantes de dicha comparación son: i) la estructura educativa de la población inmigrante no comunitaria es menos cualificada que la nativa; mientras que ii) la estructura educativa de la población inmigrante comunitaria es más cualificada que la española. Y las diferencias entre nativos e inmigrantes no procedentes de la Unión Europea son aun mayores si controlamos por edad.

Por último, es interesante mencionar el trabajo de Lacomba y Lagos (2005) donde se analiza el efecto de la inmigración sobre la decisión óptima de retiro de los nativos. También en esta cuestión, el efecto de la inmigración sobre el apoyo político a posponer la edad de jubilación depende crucialmente de la edad y del nivel de cualificacion de la cuota de inmigrantes.

\section{GRÁFICO 9}

ESTRUCTURA EDUCATIVA DE LA POBLACIÓN NATIVA E INMIGRANTE (24 A 44 AÑOS)

\section{Distribución de los hombres de nacionalidad española y extranjera entre 25 y 44 años según nivel de formación}

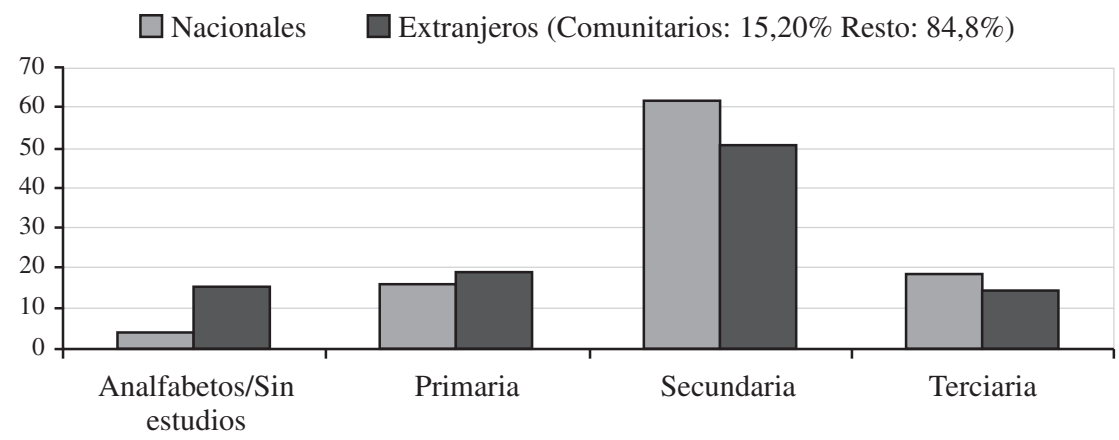

\section{Distribución de las mujeres de nacionalidad española y extranjera entre 25 y 44 años según nivel de formación}

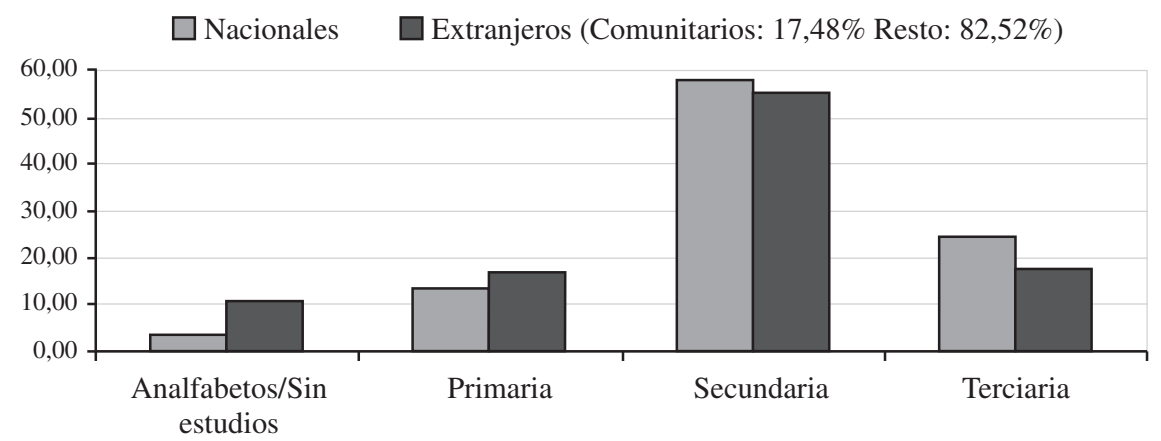




\section{GRÁFICO 10}

\section{ESTRUCTURA EDUCATIVA DE LA POBLACIÓN NATIVA E INMIGRANTE} (45 A 64 AÑOS)

\section{Distribución de los hombres de nacionalidad española y extranjera entre 45 y 64 años según nivel de formación}

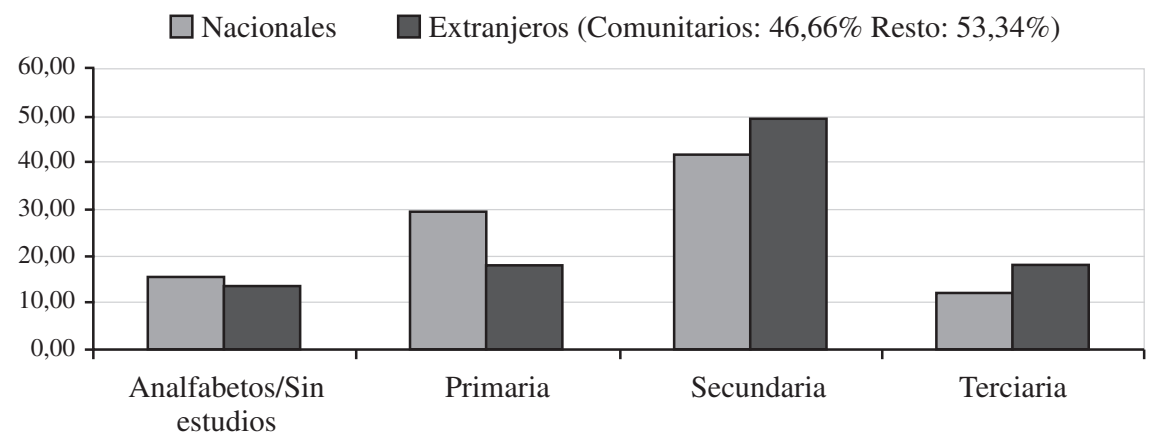

\section{Distribución de las mujeres de nacionalidad española y extranjera entre 45 y 64 años según nivel de formación}

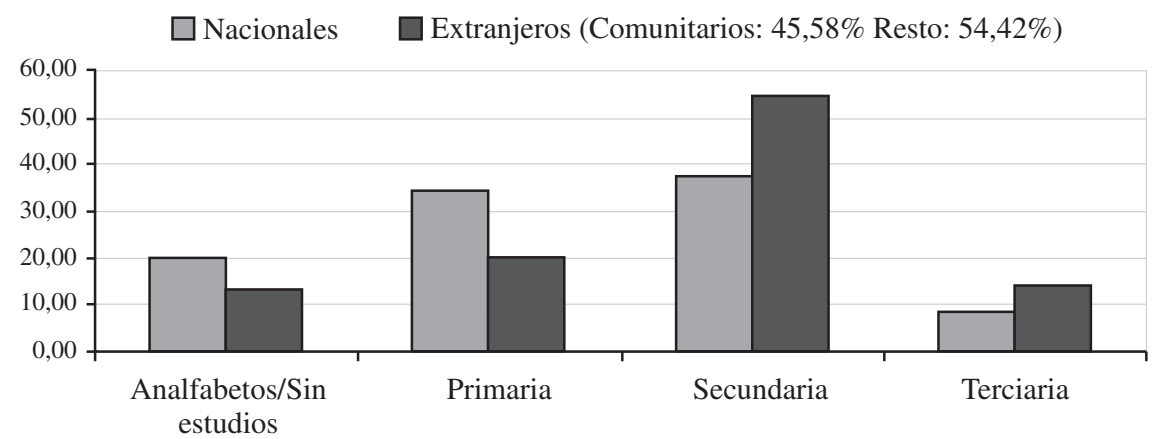

\subsection{Inmigración y Generosidad de las Pensiones}

Como vemos en la identidad contable (1) que determina el gasto en pensiones como porcentaje del PIB, el Factor Institucional depende a su vez de dos variables:

a) La primera variable, representada por la ratio entre el número de pensiones y la población mayor de 65 años depende de la elegibilidad legal para recibir una pensión. Está sujeta, por tanto, a las normas de acceso a las pensiones contributivas por parte de los inmigrantes.

b) La segunda variable institucional, es la ratio entre pensión media y productividad media (PIB por ocupado) y depende de las normas de cálculo de las pensiones y de la evolución de la productividad. Esta ratio es un indicador del grado de generosidad del sistema. Si bien es cierto, que para el caso de España, esta ratio se ha mantenido relativamente constante alrededor del 17\% (Jimeno, 2002a), su 
evolución futura dependerá, no sólo de los cambios en la productividad que experimente la economía (progreso tecnológico) sino también, lo que es más importante, de cómo se trasladen los aumentos en la productividad a las pensiones ${ }^{3}$.

La inmigración afecta al Factor Institucional por dos canales distintos: i) el canal económico a través del impacto que la inmigración tiene sobre las variables económicas determinantes (precios relativos de los factores - estructura salarial y tipos de interés-) y, por lo tanto, sobre la productividad de la economía; ii) el canal político, al influir en la decisión de voto de los ciudadanos y, consecuentemente, impulsando cambios normativos del sistema de pensiones.

\subsubsection{INMIGRACIÓN Y ELEGIBILIDAD}

Pensando únicamente en el gasto futuro en pensiones, la política más favorable consistiría en limitar el derecho de los inmigrantes a recibir una pensión. Sin embargo, esta política restrictiva no es implementable, pues el derecho a percibir una pensión se devenga automáticamente cuando se tiene una historia laboral suficientemente larga. Además, es importante resaltar que este derecho perdura a través de acuerdos internacionales de «portabilidad de pensiones» incluso si el inmigrante decide regresar a su país en el momento de la jubilación. La elegibilidad de la población inmigrante disminuye a medida que aumenta la edad del inmigrante en el momento de entrar a trabajar en el país. Así, si el inmigrante entra en el país a una edad avanzada podría suceder que al jubilarse no hubiera alcanzado la historia laboral mínima exigida para recibir una pensión contributiva (en España, por ejemplo, son 15 años). Por lo tanto, el impacto de la inmigración sobre la elegibilidad depende de las características institucionales de cada país, así como de la pirámide poblacional de la inmigración en el momento de entrar a formar parte del mercado de trabajo de dicho país. En España se observa que la población inmigrante no comunitaria entra muy joven, por lo que no debería tener ningún impacto sobre la elegibilidad futura. En todo caso, si suponemos que la tasa de empleo de los inmigrantes es superior a la de los nativos (de hecho en 2007 la tasa de empleo de los inmigrantes es 2,5 puntos superior a las de los nativos, $66,2 \%$ frente a $68,7 \%$ ), la elegibilidad futura debería aumentar.

\subsubsection{INMIGRACiÓn Y TASA DE SUSTITUCiÓn DE LAS PENSIONES}

El efecto de la inmigración sobre la ratio pensión media-productividad media es probablemente el más difícil de identificar, pues tanto la pensión media como la pro-

3 Ver Conde-Ruiz y Alonso (2006) para un estudio comparado de la evolución del factor de generosidad de distintos estudios sobre España. 
ductividad media dependen en gran medida del comportamiento del mercado de trabajo. Si revisamos la evidencia internacional sobre inmigración en los países de la OCDE podemos identificar dos hechos relevantes: i) en media, la estructura de edad de la población inmigrante es más joven que la de la población nativa; y ii) la estructura educativa de los inmigrantes es menos cualificada que la de la población nativa. Como hemos visto anteriormente la economía española cumple con estos dos hechos estilizados.

La entrada de inmigrantes poco cualificados afecta a la productividad por dos vías: por un lado, crece la productividad de los trabajadores más cualificados, pero por otro lado incrementa el porcentaje de trabajadores poco cualificados, lo que provoca una caída en su productividad y salario. En un modelo con salario flexible y factor trabajo heterogéneo, Johnson (1997) demuestra que la inmigración de baja cualificación aumenta el wage premium (diferencia entre el salario de trabajadores cualificados y no cualificados). No obstante, diferentes trabajos empíricos muestran que el efecto de la inmigración sobre los salarios relativos es positivo pero muy pequeño. Esta evidencia empírica se ha encontrado tanto para la economía de Estados Unidos (Borjas (1999), LaLonde y Topel (1997)) como para algunas economías europeas (Winter-Ebmer y Zweimuller (1999); Venturini (1999)). Casarico y Devillanova (2003) sostienen que los estudios empíricos no distinguen ningún impacto de la inmigración sobre el wage premium, porque no son capaces de recoger la respuesta endógena de la población nativa ante la entrada de inmigrantes poco cualificados: mayor inversión en capital humano. Si la entrada de inmigrantes poco cualificados, aumenta el salario de los trabajadores cualificados, un porcentaje mayor de nativos decidirá educarse más, y por lo tanto el impacto sobre los salarios relativos de equilibrio será muy bajo, tal como sostiene la evidencia empírica. Es decir, en un modelo con inversión en capital humano, la desigualdad de la renta que ocasionaría la entrada de individuos pobres, se neutraliza parcialmente en el medio plazo gracias al aumento de los trabajadores cualificados. Es fácil entender que si realizáramos el mismo análisis que Casarico y Devillanova (2003) en un modelo con crecimiento endógeno obtendríamos que la productividad media tampoco crecería en el medio plazo.

Recientemente, nuevos trabajos intentan explicar el porqué shocks de oferta de trabajo tienen poco impacto sobre los salarios de equilibrio. Ottaviano y Peri (2008) sostienen que en un análisis de corto plazo el salario cae, pero que en el medio plazo los salarios se recuperan. Este resultado se debe a que la caída en la productividad observada es un efecto de corto plazo generado por la disminución del ratio capital trabajo, mientras que en el medio plazo al incrementarse la rentabilidad del capital se dinamizará la inversión en capital productivo, con el consiguiente efecto positivo sobre la productividad y los salarios.

La segunda variable que influye en la generosidad de un sistema de pensiones de reparto es la pensión media, que también depende de las características institucionales. Encontramos dos clasificaciones diferentes de los sistemas de pensiones, dependiendo de la característica en la que nos fijemos. Si miramos al componente de redistribución intrageneracional, tenemos sistemas tipo Bismarkian, o contributivos, y sistemas tipo Beveridgean, o asistenciales. La literatura utiliza la etiqueta de «Bismar- 
kiano» para denominar a aquellos sistemas contributivos donde las pensiones dependen de la historia laboral de los individuos; mientras que utiliza la de «Beveridgiano» para definir un sistema de pensiones donde todos los individuos reciben la misma pensión independientemente de su historial laboral. En relación a los derechos devengados por las cotizaciones, se suele distinguir entre sistemas de prestación definida, en los que se garantiza una tasa de reemplazo - replacement rate- o de contribución definida, en los que se garantiza un nivel de cotización.

Haupt y Peters (1988) demuestran que los efectos de la inmigración sobre los sistemas de pensiones son claramente distintos dependiendo de las características del sistema en lo que se refiere a los derechos devengados. Así, en un sistema de contribución definida, la entrada de inmigrantes beneficia a los jubilados, pues aumenta las cotizaciones y por lo tanto permite aumentar las pensiones que se pagan en el momento de entrada. Por el contrario, en un sistema de prestación definida (que garantiza la tasa de reemplazo), la entrada de inmigrantes beneficia a los trabajadores, dado que amplía el número de contribuyentes para pagar una misma cantidad de pensiones ya comprometidas, permitiendo así reducir las cotizaciones. A diferencia de este artículo, el resto de los trabajos no tiene en cuenta esta diferencia y suponen que, o bien las contribuciones (Razin y Sadka (2000)), o bien las prestaciones permanecen fijas (Scholten y Thum (1996)). En consecuencia, podemos concluir que en un sistema de pensiones de contribución definida puro, los jubilados serán pro-inmigración, mientras que en un sistema puro de prestación definida, serán los trabajadores los que deseen que la inmigración crezca.

A este respecto, Leers, Meijdam y Verbon (2003) sostienen que incluso en los casos donde la inmigración podría soportar el coste de las pensiones (sistema de contribución definida), esto nunca ocurrirá bajo el supuesto de que la inmigración es un factor móvil. La explicación es que los inmigrantes no irán a países donde se prevea que toda la carga fiscal caerá sobre ellos para poder pagar las pensiones y preferirán emigrar a países menos afectados por el envejecimiento o donde se hayan realizado las reformas del sistema de pensiones necesarias para garantizar su sostenibilidad sin necesidad de aumentar mucho la carga impositiva.

Como ya hemos indicado, los sistema de pensiones son instituciones muy complejas que no solo redistribuyen renta entre generaciones (de jóvenes a mayores) sino que también pueden realizarlo dentro de cada generación (de ricos a pobres). Claramente, el componente de redistribución intrageneracional es muy alto en un sistema tipo Beveridgiano, mientras que un sistema tipo Bismarkiano suele ser menor. Sorprendentemente, Krieger (2003) demuestra que la conclusión respecto a la entrada de inmigrantes que obtenían Haupt y Peters (1988) al distinguir entre prestación definida y contribución definida no varía cualitativamente en un sistema tipo Beveridgiano o tipo Bismarkiano. Por último, Munz y Werding (2005) en un análisis realizado para Alemania, Italia, Reino Unido y Estados Unidos, muestran como esta componente del sistema de pensiones (Beveridge vs Bismark) es determinante para caracterizar a los inmigrantes como contribuyentes o receptores netos del sistema de pensiones. 


\section{Conclusiones}

A lo largo del artículo hemos analizado como la inmigración afecta a cada uno de los factores que componen el gasto futuro en pensiones. Considerando todos los efectos, podemos concluir lo siguiente: $i$ ) en el medio plazo, es posible contrarrestar mediante la inmigración el impacto negativo del envejecimiento poblacional: disminuir la tasa de dependencia futura con una inmigración selectiva por edad, con la premisa de que la pirámide de la población inmigrante debe ser lo más complementaria posible a la pirámide poblacional nativa; ii) no existe evidencia empírica de que la inmigración afecte al desempleo ni a la distribución salarial futura, iii) si la tasa de empleo de los inmigrantes es mayor que la de los nativos, la cobertura del sistema de pensiones tiende a aumentar, y iv) el impacto de la inmigración sobre al generosidad de las pensiones varía en función de las características institucionales particulares, y en concreto depende de si el sistema es de contribución o de prestación definida.

En conclusión, en función de las características de cada país (pirámide poblacional, estructura educacional y sistema de pensiones), puede existir una política de inmigración óptima, aunque, por si sola, no será capaz se solventar los problemas clásicos de los sistemas de pensiones de reparto y prestación definida. Sin embargo, al examinar las políticas de inmigración llevadas a cabo por los distintos países, se observa que no responden a un diseño óptimo, lo que ha generado un amplio esfuerzo investigador centrado en analizar cual es la política inmigratoria preferida por los individuos. Si pensamos en un modelo muy sencillo con tres generaciones como el analizado en la sección 2 , la entrada de inmigrantes en el periodo $t$ siempre tiene efectos positivos e inmediatos sobre el sistema de pensiones puesto que aumentan las cotizaciones (y por lo tanto la recaudación del sistema). Este resultado, sin embargo, no es concluyente pues un análisis riguroso del sistema de pensiones siempre ha de realizarse desde un punto de vista intertemporal dado que las cotizaciones devengan derechos sobre pensiones futuras (sobre todo en los sistemas de prestación definida). Los efectos negativos, llegado el caso, ocurrirían todos en el siguiente periodo $(t+1)$, cuando los inmigrantes se jubilan con sus derechos devengados y sus hijos empiezan a cotizar. En este contexto intertemporal, los trabajos desde Razin y Sadka (1999), afirman que los jubilados son siempre pro-inmigración pues reciben su pensión justamente en el periodo $t$, donde se disfrutan los efectos positivos. Pero sorprendentemente, Razin y Sadka (1999) sostienen que, en un entorno dinámico, no solo los jubilados sino todos los individuos se benefician de la inmigración, incluso cuando el nivel de cualificación de los inmigrantes es bajo. Ello se explica porque los costes netos de la inmigración se trasladan al futuro, y por este motivo el resultado de un equilibrio político será favorecer políticas de inmigración muy generosas. Krieger (2004) matiza estos resultados y sostiene que todo dependerá del entorno demográfico y económico que exista en el momento de jubilación de los inmigrantes que entraron en el país en el periodo anterior. Así, el resultado de Razin y Sadka (1999) se basa únicamente en el supuesto de que los hijos de los inmigrantes tienen la misma estructura educativa que los hijos de los nativos y por lo tanto, en términos per cápita, la situación an- 
tes de recibir los inmigrantes $t-1$ y cuando estos se jubilan $t+1$ es exactamente la misma. Por el contrario, Krieger (2003) asume que el entorno económico en $t+l$ (cuando los inmigrantes se jubilan) ha empeorado pues la estructura educacional de los hijos de los inmigrantes presenta menor cualificación que la de los nativos. La intuición es muy sencilla: los trabajadores actuales no demandarán la entrada de inmigrantes poco cualificados si anticipan que los hijos de estos (que pagarán sus pensiones) tendrán una cualificación menor que sus hijos disminuyendo, por tanto, el nivel de cualificación agregado del país y los ingresos futuros por cotizaciones. Krieger (2004) demuestra que este resultado es robusto incluso al caso donde los inmigrantes tienen pautas de fertilidad más altas que los nativos.

Esta discusión nos sirve para arrojar algo de luz sobre la afirmación planteada en la introducción de que solo generosas políticas de inmigración serán capaces de garantizar el equilibrio financiero futuro de los sistemas de pensiones de reparto con prestación definida, siempre suponiendo que la política de inmigración fuera selectiva por edad. Recordemos que la sostenibilidad financiera de los sistemas de pensiones de reparto con prestación definida parte de la premisa de que la tasa de dependencia se mantiene dentro de unos limites razonables, lo que implica que cada generación ha de ser ligeramente más numerosa que la anterior. Como hemos indicado la tasa de dependencia no solo depende de la tasa de natalidad sino también de la esperanza de vida. En ente sentido, la conclusión es clara: en el medio plazo, la entrada selectiva de inmigrantes impide que se dispare la tasa de dependencia, contribuyendo a la sostenibilidad del sistema; en el largo plazo, sin embargo, la respuesta es negativa pues una vez que se absorben los inmigrantes la situación revierte a su estado inicial. Es decir, si las pautas demográficas de los nativos consisten en tener menos hijos y mayor esperanza de vida, no existe ninguna razón para suponer que los inmigrantes no adquirirán esas mismas pautas de comportamiento, una vez integrados completamente en el país de acogida. En otras palabras, si el sistema de pensiones no es financieramente sostenible en la situación inicial, antes de la entrada de los inmigrantes, es porque, en media, el derecho a recibir una pensión es demasiado alto en relación a las cotizaciones pagadas. Si no se toma ninguna medida para solucionar este problema, en el largo plazo, tras la absorción de los inmigrantes por el país de acogida, la situación del sistema de pensiones será idéntica a la situación inicial. Es decir, la inmigración selectiva por edad únicamente afecta a la sostenibilidad financiera del sistema de pensiones en el medio plazo, pues retarda la explosión de la tasa de dependencia y traslada el problema a la siguiente generación. En conclusión, resulta evidente que la inmigración por si sola no puede garantizar el equilibrio financiero futuro de los sistemas de pensiones de los países occidentales, por lo que todos los argumentos a favor de reformar los sistemas de pensiones son igualmente relevantes, tanto en un contexto de inmigración selectiva por edad como en uno de ausencia de inmigración. Por último, a pesar de que Krieger (2005) lo analiza parcialmente, creemos que hay un aspecto de la inmigración que aun no ha sido analizado en profundidad por los expertos y que debería ser estudiado, y es en qué medida la inmigración puede ayudar en la transición hacia un sistema de pensiones mixto con una parte de reparto y otra parte de capitalización. 


\section{Referencias}

[1] AUERBACH, A. J. y L. J. KOTLIKOFF (1987): Dynamic Fiscal Policy, Cambridge, Cambridge University Press.

[2] BORJAS, G. (1994): «The Economics of Immigration», Journal of Economic Literature, Vol. XXXII (Dec) pp. 1667-1717.

[3] BORJAS, G. (1999): «The Economic Analysis of Immigration», en Handbook of Labor Economics, Volume 3, O. Ashenfelter y D. Card (eds). North-Holland.

[4] BORJAS, G. y S. J. TREJO (1991): «Immigrant participation in the Welfare System», Ind. Lab. Rel. Rev., Jan.1991, 44(2), pp. 195-211.

[5] CASARICO, A. y C. DEVILLANOVA (2003): «Social Security and migration with endogenous skill upgrading», Journal of Public Economics 87, pp. 773-797.

[6] CONDE-RUIZ J. I. y J. ALONSO (2006) «El Sistema de Pensiones en España ante el Reto del Envejecimiento», Presupuesto y Gasto Público, n. ${ }^{\circ}: 44 / 2006$, pp. 51-73.

[7] HAUPT, A. y W. PETERS (1988) «Public Pensions and voting on immigration», Public Choice 95 (3), pp. 403-413.

[8] HU, W. (1997): «Elderly Immigrants on Welfare», Journal of Human Resources 33 (3), pp. 711-741.

[9] JIMENO, J. F. (2002a): «Demografía, empleo, salarios y pensiones», Documento de Trabajo de FEDEA 2002-04, Madrid.

[10] JIMENO, J.F. (2002b): «Incentivos y desigualdad en el sistema español de pensiones contributivas de jubilación», Documento de Trabajo de FEDEA 2002-13, Madrid.

[11] JOHNSON, G.E. (1997): «Changes in earnings inequality: the role of demand shifts. Journal of Economic Perspectives 11 2, pp. 41-54.

[12] KEMNITZ, A. (2003): «Immigration, unemployment, and pensions», Scandinavian Journal of Economics, 105 (1), pp. 31-47.

[13] KRIEGER, T. (2003): «Voting on low-skill immigration under different pension regime» Public Choice 117, pp. 51-78.

[14] KRIEGER, T. (2004): «Fertility Rates and Skill Distribution in Razin and Sadka's Migration-Pension Model: A Note» Journal of Population Economics 17, pp. 177-182.

[15] KRIEGER, T (2005). Public Pensions and Immigration: A Public Policy Approach. Edward Elgar, Cheltenham.

[16] LACOMBA, J. A. and F. LAGOS (2005): «The role of immigration in the retirement age reforms: A theoretical analysis», Topics in Economic Analysis and Policy, 5(1), pp. 1- 18.

[17] LALONDE, R. J. y R. H. TOPEL (1997): «Economic Impact of international migration and the economic performance of migrants» en Handbook of Population and $\mathrm{Fa}$ mily Economics, Rosenzweig, M. and O. Stark (eds). North-Holland.

[18] LEE, R. D. y T. W. MILLER (1997): «The Future Fiscal Impact of Current Immigrants», en The New Americans, J. P. Smith and B. Edmonton (eds), Washington, DC. National Academy Press.

[19] LEERS, T., MEIJDAM, L. y VERBON (2003): «Ageing, migration and endogenous public pensions» Journal of Public Economics 88, pp. 131-159.

[20] MELTZER, A. y S. RICHARD (1981): «A Rational Theory of the Size of Government» Journal of Political Economy 89(5), pp. 914-27. 
[21] MIRRLESS, J. (1982): «Migration and Optimal Taxes» Journal of Public Economics, Vol. 18, pp. 319-342.

[22] MÜLLER T. (1999): «Migration, dual labor markets and social welfare in a small open economy», en Migration: The Controversies and the Evidence, R. Faini, J. de Melo y K. Zimmermann (eds), Cambridge, Cambridge University Press.

[23] MUNZ, S. y M. WERDING (2005): «Public Pensions and International Migration: Some Clarifications and Illustrative Results» Journal of Pension Economics and Finance, 4 (5), pp. 181-207.

[24] MUSGRAVE, R. A. (1969): Fiscal Systems, New Haven, CT: Yale University Press.

[25] OATES W. E. (1968): «The theory of public finance in a federal system» Canadian Journal of Economics, 1, pp. 37-54.

[26] OTTAVIANO, G. y G. Peri (2008) «Rethinking the Effects of Immigration on Wages» mimeo.

[27] ORTEGA, F. (2004): «Immigration and the Survival of the Welfare State». UPF Working Paper 815. Universidad Pompeu Fabra, Barcelona.

[28] RAZIN, A. y E. SADKA (1997): «Tax Burden and Migration: A political Economy Perspective», IMF Working Paper 97/78.

[29] RAZIN, A. y E. SADKA (1995): «Resisting migration: wage rigidity and income redistribution» American Economic Review, Papers and Proceedings 85 (2), pp. 312316.

[30] RAZIN, A. y E. SADKA (1999): «Migration and pension with international capital mobility», Journal of Public Economics 74, pp. 141-150.

[31] RAZIN, A. y E. SADKA (2000): «Unskilled Migration: A Burden or a Boom for the Welfare State?» Scandinavian Journal of Economics 102 (3), pp. 463-479.

[32] RAZIN, A., SADKA, E. y P. SWAGEL (2002): «Tax Burden and Migration: A political Economy theory and Evidence» Journal of Public Economics 85, pp. 167190.

[33] SCHOLTEN, U. y M. THUM (1996): «Public Pensions and immigration policy in a democracy», Public Choice 87(3), pp. 347-316.

[34] SITGLER, G. J. (1957): «The tenable range of functions of local government» Joint Economic Committee, Federal Expenditure Policy for Economic Growth and Stability, reprinted in Private Wants and Public Needs, rev. ed. (1965), ed. E.S. Phelps, New York. Norton.

[35] STORESLETTEN, K. (2000): «Sustaining Fiscal Policy through Immigration», Journal of Political Economy 108, pp. 300-323.

[36] STORESLETTEN, K. (2003): «Fiscal Implications of Immigration- A Net Present Value Calculation», Scandinavian Journal of Economics 105(3), pp. 487-506.

[37] TAMURA, Y. (2006): «Disagreement over the immigration of low-income earners in a welfare state» Journal of Population Economics, 19, pp. 691-702.

[38] VENTURINI, A. (1999): «Do immigrants working illegally reduce the natives' legal employment?», Journal of Population Economics 12(1), pp. 135-154.

[39] WINTER-EBMER, R. y J. ZWEIMULLER (1999): «Do immigrants displace young native workers: the Austrian experience», Journal of Population Economics 12 (2), pp. 327-340.

[40] WILDASIN, D.E. (1994): «Income redistribution and migration» Canadian Journal of Economics 27 (3), pp. 637-656. 

